\title{
Análise de estrutura interna da Escala Baptista de Depressão Versão Hospital-Ambulatório (EBADEP-HOSP-AMB)
}

\author{
Gabriela da Silva Cremasco \\ Makilim Nunes Baptista
}

\section{RESUMO}

A presente pesquisa teve como objetivo buscar evidências de validade baseadas na estrutura interna para a Escala Baptista de Depressão Versão Hospital-Ambulatório (EBADEP-HOSPAMB) a partir da análise fatorial exploratória e Teoria de Resposta ao Item (TRI). Participaram do estudo 210 pacientes com Doença Renal Crônica (DRC) em hemodiálise, ambos os sexos, com idades entre 18 a 82 anos $(M=53,40 ; D P=14,40)$. Com a análise fatorial exploratória 0 instrumento foi reduzido de 43 para 20 itens, em dois fatores nomeados inicialmente de "Aspectos negativos" (14 itens) e "Aspectos positivos" (6 itens), explicando 56\% da variância dos itens. Os índices de fidedignidade para o fator 1 e fator 2 da escala foram de 0,95 e 0,89, respectivamente. Pela TRI, foram verificados os parâmetros dos itens e das pessoas, sendo obtidos bons índices de ajuste para os dois fatores da EBADEP-HOSP-AMB. Conclui-se que o instrumento apresentou boas propriedades psicométricas, podendo ser uma ferramenta de rastreio de sintomatologia depressiva útil para o contexto hospitalar/ambulatorial, sendo necessários outros estudos com amostras mais representativas para realização de novas análises.

Palavras-chave: análise fatorial; teoria de resposta ao item; evidências de validade.

\section{ABSTRACT}

\section{Analysis of the internal structure of the Baptist Depression Scale Hospital- Ambulatory Version [EBADEP-HOSP-AMB]}

The present study aimed to find evidences of validity (based on the internal structure) for the Baptist Depression Scale Hospital-Ambulatory Version (EBADEP-HOSP-AMB), using exploratory factor analysis and Item Response Theory (IRT). Two hundred and ten patients with chronic renal disease (CRD) on hemodialysis, of both sexes, aged 18-82 years, participated in the study. Using exploratory factor analysis, the instrument was reduced from 43 to 20 items, in two factors initially named "Negative Aspects" (14 items) and "Positive Aspects" (6 items), which explain $56 \%$ of item variance. Reliability indexes for those factors were 0.95 and 0.89 , respectively. Using IRT, the parameters of both items and persons were verified, and good adjustment indexes were obtained for the two factors of EBADEP-HOSPAMB. We conclude that the instrument had good psychometric properties and could be a tool for screening depressive symptomatology in hospital / outpatient settings; other studies with more representative samples are necessary to carry out new analyzes.

Keywords: factor analysis; item response theory; evidence of validity.

A depressão é um transtorno mental que afeta pessoas no mundo todo, acarretando diversos prejuízos, sendo considerada como um dos principais fatores de risco para o suicídio (World Health Organization - WHO, 2017). Em se tratando da população em geral, é estimado que a depressão atinja de três a cinco por cento, enquanto, em populações clínicas (hospitalares), o número de pessoas acometidas é ainda maior, dado que o transtorno é encontrado em cinco a dez por cento dos pacientes ambulatoriais e em nove a $16 \%$ de

\section{Sobre os Autores}

G. S. C. orcid.org/000-0003-2075-8049 Universidade São Francisco (USF) - Campinas, SP gabisilva10@hotmail.com

M. N. B.

orcid.org/0000-0001-6519-254X Universidade São Francisco (USF) - Campinas, SP makilim01@gmail.com

\section{Direitos Autorais}

Este é um artigo de acesso aberto e pode ser reproduzido livremente, distribuído, transmitido ou modificado, por qualquer pessoa desde que usado sem fins comerciais. 0 trabalho é disponibilizado sob a licença Creative Commons CCBY-NC.

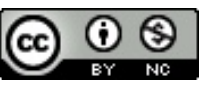




\section{- INTERACÃO EM ET PSICOLOGIA}

pacientes internados. No entanto, apesar da prevalência alta no contexto clínico, a doença é muitas vezes subdiagnosticada ou o tratamento é ofertado de forma inadequada, afetando a evolução do quadro do paciente. Assim, dos pacientes com esse transtorno de humor, apenas $35 \%$ (aproximadamente) recebem diagnóstico e tratamento adequados, o que é ainda um número muito reduzido ao se pensar nas consequências da doença (Katon, 2003; Richards, 2011).

Serafini, Budzyn e Fonseca (2017) salientam que a avaliação psicológica no cenário hospitalar possibilita a inclusão ou exclusão de hipóteses, auxiliando na formulação do diagnóstico psicológico, sendo que os testes são ferramentas que podem contribuir nesse sentido. Em uma revisão integrativa da literatura a respeito de instrumentos de depressão na população brasileira em diferentes contextos, Baptista e Borges (2016) consultaram as bases Index Psi e BVS Psi (SciELO e PePSIC) entre os anos de 2005 e 2015. Foi verificado que os instrumentos mais utilizados foram o Beck Depression Inventory (BDI), a Hamilton Depression Rating Scale for Depression (HAM-D) e a Hospital Anxiety and Depression Scale (HADS).

Dos instrumentos citados, a HADS, especificamente, é um dos mais utilizados nacional e internacionalmente no que se refere ao contexto hospitalar, que também é o foco da presente pesquisa, portanto serão apresentadas algumas considerações a respeito dessa escala. Embora a HADS seja amplamente utilizada, não se tem ainda uma concordância quanto a sua estrutura, tendo em vista que os resultados dos estudos se mostraram divergentes. A pesquisa de Razavi, Delvaux, Farvacques e Robaye (1990) indicou que a escala possui um único fator, ao passo que Hung, Bounsanga, Tang, Chen e Cheng (2015) e Bergerot, Laros e Araujo (2014) encontraram dois fatores e Rodgers, Martin, Morse, Kendell e Verril (2005) e Barth e Martin (2005) obtiveram três fatores.

Considerando as discrepâncias em relação a estrutura fatorial da HADS, Cosco, Doyle, Ward e McGee (2012) realizaram uma revisão da literatura acerca do instrumento. Foram consultadas as bases MEDLINE, ISI Web of Knowledge, CINAHL, PscyINFO e EMBASE, considerando os artigos publicados entre maio de 2000 e maio de 2010, sendo inclusos os estudos que se propuseram a avaliar a estrutura da escala. Dentre os 50 estudos avaliados, os autores verificaram que 25 indicaram uma estrutura de dois fatores, 17 revelaram três fatores, cinco revelaram-se unidimensionais, além de outros dois artigos que demonstraram uma estrutura com quatro fatores. Um dos estudos demonstrou suporte igual para dois e três fatores. Como conclusão, os autores discutiram os resultados heterogêneos obtidos, indicando que a estrutura da HADS não é clara, e pontuaram ainda que o instrumento não diferencia de forma consistente os construtos depressão e ansiedade, servindo mais como uma medida geral sobre o sofrimento emocional.

No Brasil são poucas as escalas de depressão aprovadas para a utilização por psicólogos e, mais especificamente, em ambientes hospitalares, não há uma escala desenvolvida e normatizada para a realidade brasileira. Nesse sentido, a Escala Baptista de Depressão Versão Hospital-Ambulatório (EBADEP-HOSP-AMB) (Baptista, 2013) tem como objetivo rastrear sintomas de depressão no contexto ambulatorial/ hospitalar. 0 instrumento foi construído a partir de indicadores sintomáticos de depressão (humor deprimido, perda ou diminuição de prazer, desesperança, desamparo, indecisão, sentimento de incapacidade, sentimentos de inadequação, carência/dependência, negativismo, esquiva de situações sociais, sentimento de inutilidade, autocrítica exacerbada, culpa, pensamento de morte, baixa autoestima, falta de perspectiva sobre o presente, ansiedade e irritação) presentes no Manual Diagnóstico e Estatístico dos Transtornos Mentais - DSM-IV-TR (APA, 2002), na Classificação Internacional de Doenças - CID-10 (OMS, 1993), além de descritores da teoria cognitiva (Beck, Rush, Shaw, \& Emery, 1997) e da visão comportamental da depressão (Ferster, Culbertson, \& Boren, 1977).

Por se tratar de uma ferramenta própria para o contexto hospitalar/ambulatorial, a escala foi elaborada sem a presença dos indicadores vegetativos (diminuição ou aumento do apetite, insônia ou hipersonia, agitação ou retardo psicomotor e fadiga ou perda de energia). Esses indicadores estão presentes nas demais versões da escala de mesma autoria (versão adulto e infanto-juvenil) e na maior parte dos instrumentos utilizados nesse cenário, que poderiam enviesar os dados obtidos devido à sobreposição de sintomas comuns tanto às patologias clínicas quanto à depressão, acarretando em problemas para compreender a relação do transtorno de humor com outras doenças (Baptista, 2013).

Algumas pesquisas foram realizadas com a EBADEPHOSP-AMB, tais como o estudo de Messias (2014), que teve com objetivo buscar evidências de validade baseada na relação com variáveis externas para a escala. Participaram da pesquisa 80 mulheres, com diagnóstico de fibromialgia ou câncer, com idades entre 31 e 82 anos $(M=53,86$; $D P=$ $12,68)$, que responderam ao Questionário de Dor McGill (BrMPO), ao Questionário de Saúde Geral de Goldberg (QSG) e à Escala de Percepção de Suporte Social (EPSUS). Foram encontradas correlações moderadas e positivas que variaram de $r=0,49$ a $r=0,64(p<0,05)$ entre a EBADEP-HOSP-AMB e o QSG. Estes resultados indicaram que quanto maior a 


\section{H* INTERACÃO EM 27. PSICOLOGIA}

sintomatologia de depressão, pior a percepção de saúde geral. As correlações entre a EBADEP-HOSP-AMB e a EPSUS foram moderadas e negativas entre os fatores variando de $r=$ $-0,48$ a $r=-0,62(p<0,05)$, indicando que quanto maior a sintomatologia depressiva apresentada, pior a percepção de suporte social do sujeito. Adicionalmente, as pacientes com sintomatologia depressiva apresentaram maior quantidade de pontos dolorosos no corpo, com maior frequência e intensidade de dor.

Outra pesquisa utilizando o instrumento foi o estudo de Sette, Capitão e Carvalho (2016) que teve como intuito investigar a sintomatologia depressiva em pacientes oncológicos, além de possíveis diferenças quanto ao gênero, qualidade de vida e suporte social. A amostra foi composta por 68 pacientes, na faixa etária entre 25 a 83 anos $(M=55,4$; $D P=13,3$ ), sendo $85,3 \%$ do sexo feminino. Foram aplicados um questionário sociodemográfico, a Escala de Percepção do Suporte Social - versão adulto (EPSUS-A), a Medical Outcomes Short-Form Health Survey (SF-36), bem como a EBADEP-HOSP-AMB. De forma geral, os resultados indicaram que as pessoas que declararam ter histórico de depressão pontuaram mais na EBADEP-HOSP-AMB $(M=19,39 ; D P=$ 12,64), em relação aos que indicaram não ter nenhum histórico da doença. $(M=13,69 ; D P=10,96)$. Foi verificado que as pessoas com menor percepção de suporte social também apresentaram mais sintomatologia depressiva $(t(\mathrm{gl})$ $=3,13$ (51); $p<0,01 ; d=0,77$ ).

0 único estudo que se propôs a buscar evidências de validade baseada na estrutura interna para a EBADEP-HOSP$A M B$ até o presente momento foi o de Marques (2016). A amostra foi composta de 75 pacientes com lesão encefálica, sendo $46(61,3 \%)$ do sexo masculino, com média de idade de 44,0 anos $(D P=11,4)$. Dois itens tiveram desajustes de infit e outfit (6- Tenho vontade de fazer coisas que gostava antes; e 3- Sinto que as pessoas me apoiam), e dois itens tiveram desajustes de outfit (9- Continuo a (o) mesma (o) com as pessoas; e 23- Prefiro a solidão). A análise de Teoria de Resposta ao Item (TRI) evidenciou os itens mais fáceis e mais difíceis de serem endossados. Os mais fáceis foram: 5Parece que me falta alguma coisa na vida; 29- Perco a paciência por muito pouco; 15- Está mais difícil decidir sobre as coisas; 7- Tenho chorado muito; 8- Tenho sentido vontade de chorar; 16- Consigo me virar sozinho(a); e 4- Resolvo meus problemas. Já os mais difíceis foram: 26- Morrer é a solução para os problemas; 35 - Tenho pensado em me machucar de propósito; e 28- Não gosto de mim.

Com base no exposto, ressalta-se que para um instrumento ser considerado útil para o que se propõe a avaliar, é necessário que apresente determinadas características. Assim, é imprescindível que um teste psicológico possua adequadas propriedades psicométricas, ou seja, estudos de evidências de validade e confiabilidade (Adánez, 1999; Urbina, 2007). Dentre as evidências de validade propostas pela AERA, APA, e NCME (2014), o objetivo do presente estudo foi buscar evidências de validade baseada na estrutura interna para a EBADEP-HOSP-AMB.

\section{MÉTODO}

\section{PARTICIPANTES}

Foram participantes do estudo 210 pacientes com Doença Renal Crônica (DRC) em tratamento de hemodiálise, sendo $112(53,3 \%)$ do sexo masculino, com idades entre 18 e 82 anos $(M=53,40 ; D P=14,40)$, com tempo de tratamento entre 1 mês e 336 meses, o equivalente a 28 anos $(M=56,19$; $D P=64,99)$. Em relação à escolaridade, $3,8 \%$ se declararam analfabetos, $23,8 \%$ com fundamental incompleto, $32,9 \%$ com fundamental completo, $5,2 \%$ com ensino médio incompleto, $24,3 \%$ ensino médio completo, $1,4 \%$ superior incompleto e $8,6 \%$ superior completo. A amostra foi proveniente de instituições de saúde públicas e privadas de cidades do interior do estado de São Paulo e os sujeitos escolhidos por conveniência.

\section{INSTRUMENTO}

Escala Baptista de Depressão (Versão HospitalAmbulatório) (EBADEP-HOSP-AMB; Baptista, 2013): composta inicialmente de 43 itens, em escala do tipo Likert de três pontos ( 0 = sim; 1 = às vezes e 2 = não), com mínima de zero e máxima de 86 pontos, em que quanto menor a pontuação, menor a sintomatologia depressiva apresentada pelo sujeito. 0 participante deve responder ao instrumento se baseando em seus sentimentos de, no mínimo, duas semanas. Foram realizados estudos da EBADEP-HOSP-AMB com amostras de pacientes oncológicos (Sette et al., 2016), fibromiálgicos e oncológicos (Messias, 2014) e lesão encefálica (Marques, 2016).

\section{PROCEDIMENTOS}

O projeto foi aprovado pelo Comitê de Ética em Pesquisa em pesquisa da Universidade São Francisco, CAAE n. 59264816.7.0000.5514 e os participantes concordaram em participar do estudo por meio da assinatura do Termo de Consentimento Livre e Esclarecido (TCLE), impresso em duas vias, que continham as informações referentes ao estudo. As aplicações ocorreram de forma individual durante as sessões de hemodiálise com duração aproximada de 30 minutos. 


\section{W INERACÄOEM ET PSICOLOGIA}

As análises foram realizadas pelos programas FACTOR (versão 10.5.01) (Lorenzo-Seva \& Ferrando, 2006) e Winsteps (versão 3.70.0) (Linacre, 2010). A estrutura interna da EBADEP-HOSP-AMB foi verificada por meio da análise fatorial exploratória, com método de estimação Unweighted Least Squares (ULS), a partir de uma matriz de correlações policóricas por se tratar de escalas do tipo Likert. Para avaliar a dimensionalidade da escala foram utilizados os métodos de retenção Minimum Average Partial (MAP) (Velicer, 1976), HULL (Lorenzo-Seva, Timmerman, \& Kiers, 2011) e análise paralela com permutação de valores (Timmerman, \& LorenzoSeva, 2011), com rotação oblíqua direct oblimin. O índice Kaiser-Meyer-Olkin (KMO) e o teste de esfericidade de Bartlett foram utilizados para verificar se os dados eram passíveis de análise fatorial e a consistência interna foi calculada por meio do alfa de Cronbach.

Os dados foram analisados pela Teoria de Resposta ao Item (TRI) com modelo de resposta gradual (Rating Scale Model). Foi considerado como sendo ideal itens que apresentaram valores acima de 0,30 na correlação item-total (Linacre \& Wright, 1991). Já o ajuste dos itens, avaliados por meio das medidas de infit e outfit foram classificados como adequados quando dentro do intervalo entre 0,60 e 1,40 (Linacre, 2010).

\section{RESULTADOS}

Antes da realização da análise fatorial, foram excluídos um total de oito itens $(3,9,11,15,16,17,19$ e 32) pelo autor do instrumento, que em uma nova análise qualitativa da escala optou por tirar os itens que não eram considerados adequados para avaliar o descritor proposto e/ou a escrita ainda causava dúvidas aos respondentes, além do que o objetivo era a diminuição do número de itens da escala (ex: Minhas atitudes me parecem normais). A validade aparente (face validity) corresponde à revisão dos conteúdos de um teste para verificar se eles são adequados, conforme indicado por Pasquali (2007). Além disso, alguns destes itens sugeriam pertencer ao descritor vegetativo/somático (ex: Tenho dificuldades para dormir), que tendem a enviesar a pontuação da escala final e aumentam a possibilidade de falsos positivos, já que pacientes hospitalizados acabam por pontuar tais itens em detrimento da condição médica.

Assim, a análise propriamente dita da EBADEP-HOSPAMB foi realizada com 35 itens. Os métodos de retenção MAP e análise paralela recomendaram a retenção de até dois fatores e o HULL recomendou um fator. Em função disso, foram testados modelos de um e dois fatores, sendo encontrado um melhor ajuste para o modelo de dois fatores. O Kaiser-Meyer-Olkin (KMO) e o teste de esfericidade de Bartlett indicaram que os dados eram passíveis de análise fatorial (KMO $=0,93, x 2=2038,7, p<0,001)$. Ao se fixar um número de 2 fatores, 15 itens apresentaram cargas cruzadas, ou não carregaram em nenhum fator, o que resultou em uma versão com 20 itens, sendo utilizado como critério para inclusão do item uma carga fatorial mínima de 0,30. A análise dos 20 itens indicou um índice de ajuste Global Fit Index (GFI) $=0,98$, sendo explicados $56 \%$ da variância total dos itens [eigenvalues $=8,78(44 \%) ; 2,36(12 \%)$, com comunalidades entre 0,29 e 0,81 . A distribuição dos itens nos fatores é apresentada na Tabela 1.

Tabela 1. Análise Fatorial Exploratória ULS com correlações policóricas

\begin{tabular}{lccc}
\hline \multicolumn{1}{c}{ Descritores } & $\mathrm{F} 1$ & $\mathrm{~F} 2$ & $\mathrm{H} 2$ \\
\hline 01. Solidão & 0,67 & & 0,45 \\
05. Incompletude & 0,53 & & 0,34 \\
07. Choro & 0,77 & & 0,48 \\
08. Choro & 0,73 & & 0,48 \\
13. Humor & 0,83 & & 0,65 \\
14. Autoestima/Autoconceito & 0,82 & & 0,70 \\
24. Humor & 0,91 & & 0,81 \\
30. Desesperança & 0,55 & & 0,40 \\
33. Humor & 0,74 & & 0,72 \\
34. Irritabilidade & 0,52 & & 0,29 \\
36. Ideação Suicida & 0,55 & & 0,43 \\
40. Ideação Suicida & 0,59 & & 0,41 \\
41. Anedonia & 0,56 & & 0,40 \\
43. Incapacidade & 0,53 & & 0,44 \\
02. Autoestima/Autoconceito & & 0,59 & 0,34 \\
18. Humor & & 0,68 & 0,52 \\
21. Concentração & & 0,55 & 0,46 \\
27. Visão de mundo (Tríade & & 0,82 & 0,65 \\
Cognitiva) & & & \\
28. Autoestima/Autoconceito & & 0,83 & 0,66 \\
42. Anedonia & & 0,66 & 0,66 \\
Eigenvalues & 8,78 & 2,36 & - \\
Variância explicada & $44 \%$ & $12 \%$ & - \\
\hline
\end{tabular}

A correlação entre os fatores foi de 0,46 , tratando-se de uma correlação moderada conforme os critérios propostos por Dancey e Reidy (2006) e todos os itens tiveram cargas fatoriais acima de 0,50. Os coeficientes de fidedignidades dos fatores rotacionados foram de 0,95 para o fator 1 e de 0,89 para o fator 2. Conforme recomendado por George e Mallery (2003), os valores do alfa de Cronbach são: $a>0,90=$ excelente; $a>0,80=$ bom; $a>0,70=$ aceitável; $a>0,60=$ questionável; $a>0,50=$ pobre; e $a<0,50=$ inaceitável. Desse modo, a EBADEP-HOSP-AMB apresentou confiabilidade excelente e boa para o primeiro e o segundo fator, respectivamente. 0 primeiro fator ficou composto de 14 itens referentes a sintomas da depressão, tais como, choro, desesperança, irritabilidade, anedonia, dentre outros, sendo denominado inicialmente de "Aspectos negativos". O segundo fator, com os seis itens restantes, se refere a 


\section{H NTERAC̄OOEM ITH PSICOLOGIA}

aspectos que vão de encontro à sintomatologia depressiva, tais como, felicidade, prazer na realização de atividades, autoestima, dentre outros, sendo nomeado inicialmente de "Aspectos positivos".

Posteriormente, os dados foram analisados pela TRI, a partir do modelo de Rasch. Serão apresentadas as estatísticas descritivas sumarizadas referentes ao traço latente (theta), dificuldade dos itens (b), além dos índices de infit e outfit e fidedignidade de cada fator. Os resultados podem ser observados na Tabela 2 .

Tabela 2. Estatisticas descritivas sumarizadas das pessoas e dos itens

\begin{tabular}{|c|c|c|c|c|c|c|c|c|}
\hline \multirow[b]{2}{*}{ Fator } & \multirow[b]{2}{*}{ Medidas } & \multicolumn{3}{|c|}{ Pessoas } & \multicolumn{3}{|c|}{ Itens } & \multirow[b]{2}{*}{ Fidedignidade } \\
\hline & & Theta & Infit & Outfit & $b$ & Infit & Outfit & \\
\hline \multirow[t]{4}{*}{$\mathrm{F} 1$} & Média & $-0,27$ & 1,01 & 1,02 & 0,00 & 1,00 & 1,02 & 0,92 \\
\hline & DP & 1,17 & 0,32 & 0,62 & 0,56 & 0,18 & 0,27 & \\
\hline & Máximo & 2,44 & 1,90 & 4,57 & 1,26 & 1,21 & 1,40 & \\
\hline & Mínimo & $-2,41$ & 0,32 & 0,15 & $-0,97$ & 0,60 & 0,53 & \\
\hline \multirow[t]{4}{*}{ F2 } & Média & $-1,15$ & 0,99 & 0,91 & 0,00 & 1,06 & 0,91 & 0,82 \\
\hline & DP & 1,09 & 0,54 & 0,68 & 0,94 & 0,15 & 0,21 & \\
\hline & Máximo & 2,41 & 2,42 & 2,99 & 1,50 & 1,24 & 1,30 & \\
\hline & Mínimo & $-2,36$ & 0,12 & 0,14 & $-1,23$ & 0,83 & 0,61 & \\
\hline
\end{tabular}

dificuldade (b) e correlação item-total. Os resultados referentes ao fator 1 são apresentados na Tabela 3 e os do fator 2 indicados na Tabela 4.

Os dados da Tabela 3 demonstram que os valores de infit e outfit estiveram dentro do intervalo de 0,60 a 1,40 (Linacre, 2010) com exceção do item 24 (Tenho me sentido angustiado(a)) que apresentou outfit abaixo do esperado.
Como mostra a Tabela 2, a média e desvio padrão de theta dos respondentes no fator 1 ficou próxima a média dos itens, sugerindo que os itens tendem a ser ajustados para essas pessoas. Já no fator 2 , a média de theta ficou pouco mais de um logit abaixo da média dos itens, indicando que os itens desse fator foram difíceis para os respondentes. A fidedignidade do fator 1 foi considerada excelente e a do fator 2 considerada boa. A média dos valores de infit e outfit ficaram dentro do dentro do limite esperado de 0,60 a 1,40 (Linacre, 2010). Foi realizada uma análise dos itens que constituem os dois fatores quanto aos índices de infit e outfit,

Verificou-se que os itens mais fáceis foram o 5 (Parece que me falta alguma coisa na vida) e o 41 (As coisas me agradam menos do que antigamente) que apresentaram valores de $b$ igual a $-0,97$ e $-0,69$, respectivamente. Já os itens mais difíceis foram o 36 (Tenho pensado mais sobre morte) e o 7 (Tenho chorado muito) com dificuldade igual a 1,26 e 0,54, respectivamente. Em relação as correlações item-total nenhum dos itens apresentou cargas inferiores a 0,30. Foram

Tabela 3. Análise de Teoria de Resposta ao Item do fator 1

\begin{tabular}{ccccccc}
\hline Item & $\begin{array}{c}\text { Escore } \\
\text { bruto }\end{array}$ & $\mathrm{N}$ & $\begin{array}{c}\text { Dificuldade } \\
\text { (b) }\end{array}$ & Infit & Outfit & $\begin{array}{c}\text { Correlação } \\
\text { Item-Total }\end{array}$ \\
\hline 01 & 144 & 210 & 0,20 & 1,12 & 1,24 & 0,64 \\
05 & 245 & 210 & $-0,97$ & 1,13 & 1,32 & 0,66 \\
07 & 118 & 210 & 0,54 & 1,08 & 1,38 & 0,62 \\
08 & 198 & 210 & $-0,44$ & 0,98 & 0,96 & 0,69 \\
13 & 176 & 210 & $-0,19$ & 0,81 & 0,89 & 0,72 \\
14 & 133 & 210 & 0,34 & 0,80 & 0,66 & 0,71 \\
24 & 163 & 210 & $-0,03$ & 0,60 & 0,53 & 0,77 \\
30 & 125 & 210 & 0,44 & 1,13 & 1,28 & 0,63 \\
33 & 141 & 210 & 0,23 & 0,77 & 0,61 & 0,72 \\
34 & 148 & 210 & 0,15 & 1,21 & 1,40 & 0,61 \\
36 & 72 & 210 & 1,26 & 1,07 & 0,96 & 0,57 \\
40 & 187 & 210 & $-0,31$ & 1,13 & 1,08 & 0,67 \\
41 & 220 & 210 & $-0,69$ & 1,10 & 1,06 & 0,68 \\
43 & 205 & 210 & $-0,52$ & 1,12 & 0,96 & 0,67 \\
Média & 162,5 & 210,0 & 0,00 & 1,00 & 1,02 & - \\
DP & 44,3 & 0,00 & 0,56 & 0,18 & 0,27 & - \\
\hline
\end{tabular}




\section{H NTERAC̄OOEM ET PSICOLOGIA}

obtidas correlações moderadas a altas com valores entre 0,57 a 0,77 .

Como pode ser observado na Tabela 4, todos os itens do fator 2 apresentaram índices de infit e outfit dentro do intervalo (Linacre, 2010). Verificou-se que os itens mais fáceis foram o 18 (Sinto-me feliz com a minha vida) e o 21
(Consigo me concentrar nas atividades), com valores de dificuldade igual a $-1,23$ e - 0,76 . Os itens 28 (Gosto de mim) e 27 (Gosto da minha vida) foram os mais difíceis, com b igual 1,50 e 0,56, respectivamente. É importante frisar que a EBADEP-HOSP-AMB não avalia habilidade, ou seja, os itens considerados difíceis são aqueles com menor frequência de respostas. Foram verificadas correlações com magnitudes

Tabela 4. Análise de Teoria de Resposta ao Item do fator 2

\begin{tabular}{ccccccc}
\hline Item & $\begin{array}{c}\text { Escore } \\
\text { bruto }\end{array}$ & $\mathbf{N}$ & $\begin{array}{c}\text { Dificuldade } \\
\text { (b) }\end{array}$ & Infit & Outfit & $\begin{array}{c}\text { Correlação } \\
\text { Item-Total }\end{array}$ \\
\hline 02 & 87 & 210 & $-0,61$ & 1,24 & 1,30 & 0,71 \\
18 & 117 & 210 & $-1,23$ & 0,83 & 0,82 & 0,80 \\
21 & 94 & 210 & $-0,76$ & 0,99 & 0,99 & 0,75 \\
27 & 45 & 210 & 0,57 & 0,99 & 0,85 & 0,70 \\
28 & 26 & 210 & 1,50 & 1,23 & 0,61 & 0,62 \\
42 & 46 & 210 & 0,56 & 1,11 & 0,92 & 0,68 \\
Média & 69,2 & 210 & 0,00 & 1,06 & 0,91 & - \\
DP & 32,2 & 0,00 & 0,94 & 0,15 & 0,21 & - \\
\hline
\end{tabular}

moderadas a fortes que variaram entre 0,62 e 0,80. Posteriormente, foi feita uma análise das categorias de pontuação na escala Likert (0 a 2) da EBADEP-HOSP-AMB, como mostra a Tabela 5.

\section{Tabela 5. Categorias de respostas da} EBADEP-HOSP-AMB

\begin{tabular}{ccccc}
\hline Fator & $\begin{array}{c}\text { Categoria de } \\
\text { resposta }\end{array}$ & $\%$ & $\begin{array}{c}\text { Média } \\
\text { observada }\end{array}$ & Limiar \\
\hline Fator 1 & 0 & 56 & $-1,06$ & $-1,24$ \\
& 1 & 11 & $-0,24$ & 0,00 \\
Fator 2 & 2 & 33 & 0,82 & 1,24 \\
& 0 & 78 & $-1,83$ & $-1,58$ \\
& 1 & 11 & $-0,54$ & 0,00 \\
& 2 & 11 & 0,54 & 1,58 \\
\hline
\end{tabular}

Verificou-se que, à medida que as opções do instrumento aumentavam de valor (de 0 a 2), aumentaram também as médias observadas dos níveis de theta, ou seja, do nível de sintomatologia associadas à probabilidade da pessoa em endossar cada uma das possibilidades de resposta. A Figura 1 representa as categorias de pontuação, sendo o primeiro mapa referente ao fator 1 e o segundo mapa correspondente ao fator 2 .

A Figura 1 indica a probabilidade de endosso a uma categoria de resposta. A intersecção entre duas categorias se refere ao valor do limiar (threshold) estimado dessas categorias. No mapa correspondente ao fator 1 , observa-se que o limiar de transição da categoria 0 para a categoria 1 equivale ao valor de 0,74 de theta e da categoria 1 para a categoria 2 equivale a $-0,74$. Observa-se que a categoria 1 é pouco representativa, não se distinguindo, ao contrário das categorias 0 e 2. Já no mapa referente ao fator 2 , o limiar da categoria 0 para 1 corresponde ao valor $-0,14$ de theta e da categoria 1 para a categoria 2 equivale a 0,14 . Verificou-se que no fator 2 a categoria de resposta 1 foi um pouco mais representativa em relação a essa mesma categoria no fator 1. Já as categorias extremas $\left(\begin{array}{lll}0 & \text { e } & 2\end{array}\right)$ foram bem representativas em ambos os fatores. A seguir, são apresentados os mapas de itens, que indicam a distribuição do nível do traço latente das pessoas, além da dificuldade dos itens. 0 mapa de itens do fator 1 e do fator 2 são representados na Figura 2.

Referente ao primeiro mapa da Figura 2, correspondente ao fator 1 , observa-se que a maior parte das pessoas se agruparam entre -3 e 2 , sendo que cada \# equivale a duas pessoas, ao passo que os itens ficaram entre -1 e 1 . Em relação a distribuição dos itens na escala theta, verifica-se que alguns respondentes apresentaram um nível de theta mais alto que a dificuldade do item mais difícil da escala. Apesar de existirem lacunas entre os itens e as pessoas, observa-se que a média das pessoas se localizou próxima da média dos itens no fator. Como já relatado, o item 5 foi o mais fácil, enquanto que o 36 o mais difícil.

0 segundo mapa de itens referente ao fator 2 indica que, mesmo com a variação da escala aproximadamente entre -3 e 3 , a maior parte das pessoas ficaram agrupadas entre -3 e -1 , em que \# equivale a 8 pessoas, enquanto que os itens se agruparam entre -1 e 1 . Assim, pode-se observar que alguns itens foram difíceis de serem endossados pelos respondentes, apresentando uma dificuldade média dos itens alta em relação ao nível médio de theta. Ressalta-se que o item mais difícil foi o 28 e o mais fácil o 18 . 


\section{W"INTERACÃO EM ET PSICOLOGIA}

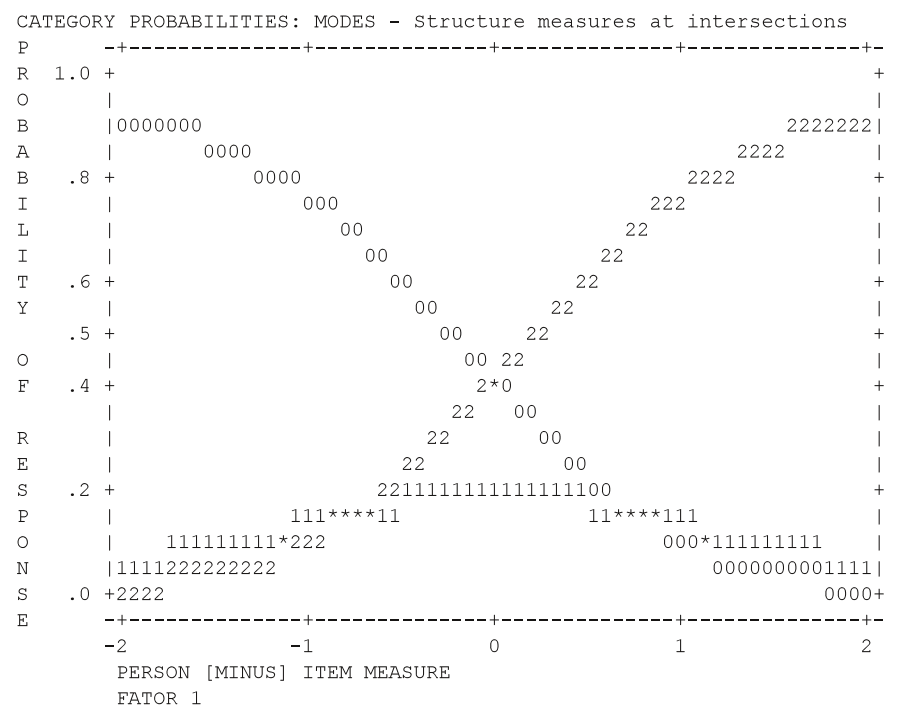

Figura 1. Probabilidade de resposta para o fator 1 e para o fator 2

\section{DISCUSSÃO}

A presente pesquisa teve como objetivos verificar a estrutura interna da EBADEP-HOSP-AMB com base na análise fatorial exploratória e TRI, a partir do modelo de resposta gradual. Os resultados da análise fatorial exploratória Unweighted Least Squares com correlações policóricas indicaram para a manutenção de 20 itens dos 35 analisados em dois fatores, visto que oito itens foram excluídos inicialmente pelo autor do instrumento por não serem adequados, sendo o fator 1 , intitulado inicialmente de Aspectos negativos composto por 14 itens e o fator 2, chamado de Aspectos positivos pelos outros seis. As cargas fatoriais dos itens apresentaram magnitudes moderadas a altas em ambos fatores.

Como já relatado, os itens da EBADEP-HOSP-AMB foram baseados em indicadores de manuais diagnósticos e teorias da depressão (APA, 2002; Beck et al., 1997; Ferster et al., 1977; OMS, 1993). A respeito disso, os instrumentos de depressão podem variar em termos da quantidade de itens para cada categoria de sintomas. Dentre essas possibilidades, têm-se as categorias propostas por Thompson (1989), que são: humor, que corresponde a tristeza, anedonia, choro, consistindo em uma categoria essencial para avaliação da depressão; sociais, que se referem a apatia, dificuldade na realização de tarefas e isolamento; cognitivos, que equivalem a desesperança, culpa, desamparo, indecisão, ideação suicida; vegetativos/ somáticos, que correspondem a alterações no sono, apetite, peso, fadiga; motores, referentes a inibição, agitação ou retardo; ansiedade; e irritabilidade.

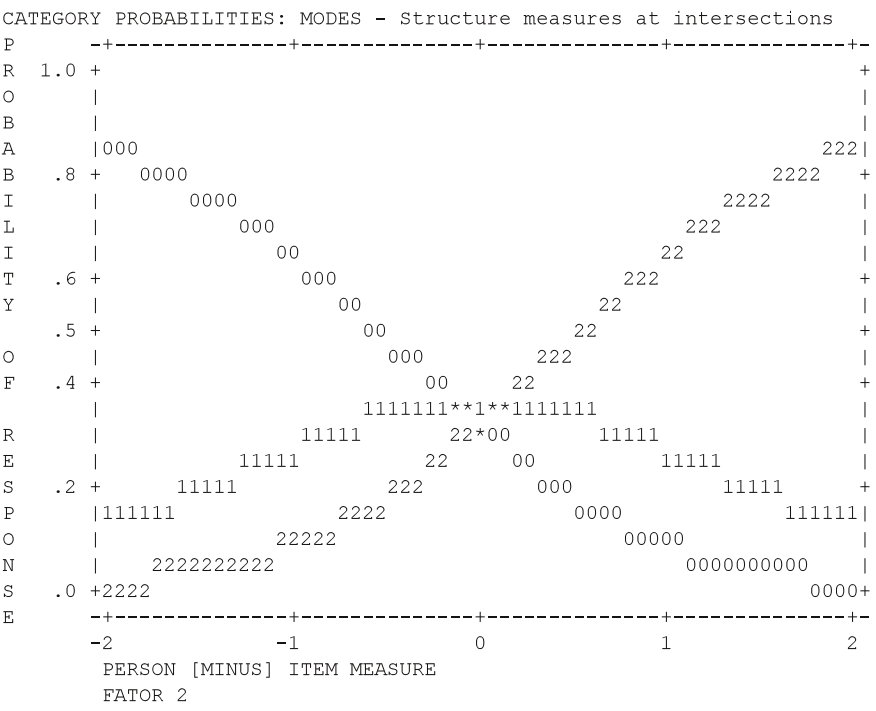

Para o desenvolvimento da EBADEP-HOSP-AMB não foram incluídos itens em relação a sintomas da depressão que poderiam se sobrepor à condição clínica do paciente. Assim, o fator 1 , incluiu itens referentes às categorias humor, cognitivos e irritabilidade. Já o fator 2 ficou composto de itens referentes às categorias humor e cognitivos. 0 item 21 (Consigo me concentrar nas atividades) corresponde a categoria sintomas cognitivos segundo Calil e Pires (1998), psíquicos conforme Del Porto (1999) ou concentração com base em Santor, Gregus e Welch (2006), sendo que no presente estudo, o item foi classificado como cognitivo. Assim, como indicado por Calil e Pires (1998), o processo de construção dos itens é influenciado pela categorização de sintomas utilizada por determinado autor, e existem divergências entre pesquisadores quanto a inserção dos sintomas em determinadas categorias.

As análises de TRI demonstraram índices de infit e outfit adequados para os itens dos dois fatores, com exceção ao outfit de um dos itens que ficou fora do intervalo (Linacre, 2010). Outro aspecto avaliado pelo modelo diz respeito à correlação item-total, sendo que na presente pesquisa foram obtidos valores moderados a fortes para todos os itens da escala (Linacre \& Wright, 1991). Marques (2016) observou, em seu estudo com amostra de pacientes com lesão encefálica, que o item 5 (Parece que me falta alguma coisa na vida) foi considerado um dos mais fáceis, enquanto que o item 28 (Não gosto de mim) foi um dos itens mais difíceis. No presente estudo, foram observados resultados semelhantes, tendo em vista que o item 5 foi o item mais fácil em relação ao fator 1 , ao passo que item 28 foi considerado o mais difícil no fator 2 . 


\section{N"INTERACÃO EM FT PSICOLOGIA}

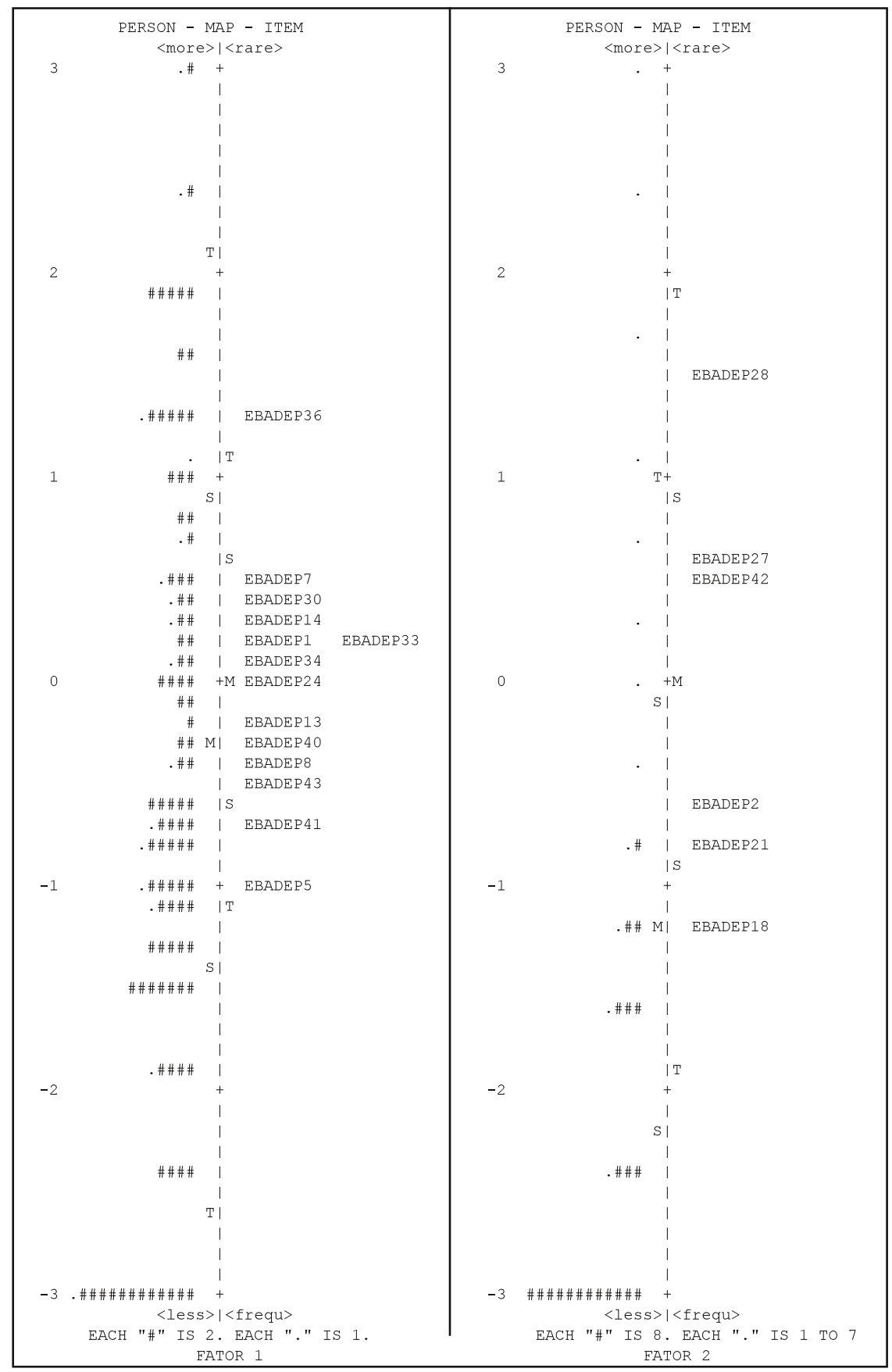

Figura 2. Mapa de pessoas e itens do fator 1 e do fator 2

No que diz respeito à fidedignidade, comparou-se os valores obtidos pelo alfa de Cronbach e pelo modelo de Rasch para ambos os fatores. Como é possível observar nos resultados apresentados, os valores de fidedignidade a partir da Teoria de Resposta ao Item (TRI) são um pouco mais baixos comparados aos obtidos a partir da Teoria Clássica dos Testes (TCT). Isso ocorre porque no modelo de Rasch é possível existir diferentes índices de precisão local na escala, ou seja, a precisão pelo modelo de Rasch se refere à média das precisões locais, gerando uma taxa menor de resíduos e, portanto, uma precisão menor e mais real, ao passo que na teoria clássica, tem-se um único índice (Linacre, 2010). 


\section{H. INTERACÃO EM LF PSICOLOGIA}

Independente do modelo utilizado, a fidedignidade se mostrou excelente para o fator 1 e boa para o fator 2 (George \& Mallery, 2003). Ainda sobre a fidedignidade, os valores obtidos para a EBADEP-HOSP-AMB foram semelhantes aos encontrados por Bergerot et al. (2014) que avaliaram a estrutura interna da HADS, um dos principais instrumentos de avaliação de depressão e ansiedade no âmbito hospitalar e ambulatorial, em que foi encontrado um alfa de 0,84 para a subescala de depressão. A variância explicada obtida no presente estudo foi de $56 \%$. No estudo de Barth e Martin (2005) com a HADS a variância explicada foi de $67 \%$.

As curvas de probabilidade possibilitam verificar se as pessoas que responderam aos itens estão utilizando a escala da forma esperada pelo pesquisador (Elliott et al., 2006). Na presente pesquisa, foi utilizada uma escala Likert de três pontos (com variação de 0 a 2). A curva 1 (às vezes) se mostrou inadequada no fator 1 , indicando uma ausência de uma região distinta na escala de theta. Já no fator 2 , a curva de resposta 1 foi mais representativa, enquanto que as categorias 0 e 2 foram bem discriminativas para ambos os fatores. Assim, os mapas correspondentes aos fatores 1 e 2 indicam que uma escala dicotômica seria suficiente para responder ao instrumento. Portanto, torna-se necessária a realização de outros estudos que verifiquem a curva de probabilidade.

A média de theta dos respondentes nos fatores da escala ficou abaixo da média de dificuldade dos itens, indicando que os itens foram difíceis de serem endossados pela amostra. É importante ponderar que a amostra foi composta por pacientes com doença renal crônica que, em sua maioria não possuem diagnóstico psiquiátrico, sendo esperado, portanto, que os itens apresentassem uma média de dificuldade maior que a média de theta das pessoas. A média de theta para o fator 1 Aspectos negativos, mesmo que inferior, ficou mais próxima da média de dificuldade do item, enquanto que em relação ao fator 2 Aspectos positivos, a média de theta ficou abaixo da média da dificuldade, o que demonstra que, para a amostra em questão, os itens do fator 2 , que avaliam os aspectos contrários à depressão, foram mais difíceis do que os do fator 1 , que avaliam os sintomas do transtorno. Desse modo, observa-se que, como relatado por Katon (2003), pessoas em tratamento ambulatorial, no caso a presente pesquisa composta por pacientes com doença renal crônica em hemodiálise, tendem a apresentar maior sintomatologia depressiva em comparação com a população geral; talvez por isso a média das pessoas, no fator 1 , tenha se aproximado da média dos itens.

A EBADEP-HOSP-AMB é um instrumento construído no Brasil e as evidências obtidas até o presente momento têm se mostrado satisfatórias. Trata-se de uma ferramenta de aplicação rápida, com itens redigidos de forma simples, possibilitando a compreensão de pessoas com pouca escolaridade. Já foram obtidas algumas evidências de validade para o instrumento, a partir de pesquisas realizadas com amostras de pacientes com lesão encefálica, oncológicos e fibromiálgicos (Marques, 2016; Messias, 2014; Sette et al., 2016)

Tendo em vista que foi encontrada uma solução com dois fatores para a EBADEP-HOSP-AMB no presente estudo, é importante realizar novos estudos com objetivo de averiguar a possibilidade de um viés semântico, pois a escala pode ser unifatorial. As análises de dimensionalidade de instrumentos com uma parcela dos itens escritos de forma positiva e outros de forma negativa têm demonstrado estruturas fatoriais diferentes, sendo muitas associadas mais pela junção dos itens pelo modo que foram redigidos e não relacionada à faceta do construto avaliado, o que é conhecido como method effects (Lindwall et al., 2012). Portanto, para estudos futuros recomenda-se a utilização de análises que testem esse tipo de viés. Conclui-se que os resultados obtidos no que concerne à EBADEP-HOSP-AMB na presente pesquisa foram satisfatórios, sendo verificadas propriedades psicométricas adequadas para a escala, tanto em relação à análise fatorial exploratória, quanto pela TRI.

\section{DECLARAÇÃO DE FINANCIAMENTO}

A pesquisa relatada foi financiada parcialmente pela bolsa de mestrado da primeira autora (CAPES) e pela bolsa de produtividade em pesquisa do segundo autor (CNPq).

\section{DECLARAÇÃO DA CONTRIBUIÇÃO DOS AUTORES}

G.S.C e M.N.B. contribuíram para a conceitualização, investigação e visualização do artigo; G.S.C e M.N.B. foram responsáveis pela obtenção de financiamento; G.S.C. fez a redação inicial do artigo (rascunho) e G.S.C e M.N.B. são os responsáveis pela redação final (revisão e edição).

\section{DECLARAÇÃO DE CONFLITOS DE INTERESSE}

Os autores declaram que não há conflitos de interesse no manuscrito submetido.

\section{REFERÊNCIAS}

Adánez, G. A. (1999). Procedimientos de construcción y análisis de tests psicometricos. Em S. M. Wechesler, \& R. S. L. Guzzo. Avaliação Psicológica: Perspectiva Internacional (pp. 57-100). São Paulo: Casa do Psicólogo. 


\section{H NTERAC̄OAEM ET PSICOLOGIA}

American Educational Research Association, American Psychological Association, National Council on Measurement in Education (2014). Standards for Educational and Psychological Testing. Washington, DC: American Psychologichal Association.

American Psychiatric Association. (2002). Diagnostic and Statistical Manual of Mental Disorders DSM-IV-TR. (4a ed.). Porto Alegre: Artmed.

Baptista, M. N. (2013). Escala Baptista de Depressão (Versão Ambulatório) - EBADEP-HOSP-AMB. Relatório Técnico não publicado. Programa de Pós-Graduação Stricto-Sensu em Psicologia da Universidade São Francisco. Itatiba. São Paulo.

Baptista, M. N., \& Borges, L. (2016). Revisão integrativa de instrumentos de depressão em crianças/adolescentes e adultos na população brasileira. Avaliação Psicológica, 15 (n. esp), 19-32. https://dx.doi.org/10.15689/ap.2016.15ee. 03

Barth, J., \& Martin, C. R. (2005). Factor structure of the Hospital Anxiety and Depression Scale (HADS) in German coronary heart disease patients. Health Qual Life Outcomes, 3(15), 1-9. http://dx.doi.org/10.1186/14777525-3-15

Beck, A. T., Rush, A. J, Shaw, F. B., \& Emery, E. (1997). Terapia cognitiva da depressão. Porto Alegre: Artes Médicas.

Bergerot, C. D., Laros, J. A., \& Araujo, T. C. C. (2014). Avaliação de ansiedade e depressão em pacientes oncológicos: comparação psicométrica. Psico-USF, 19(2), 187-197. http://dx.doi.org/10.1590/1413-82712014019002004

Calil, H. M., \& Pires, M. L. N. (1998). Aspectos gerais das escalas de avaliação de depressão. Revista de Psiquiatria Clínica, 25(5), 240-244. Recuperado de http:// www.hcnet.usp.br/ipq/revista/vol25/n5/depre255a.htm

Cosco, T. D., Doyle, F., Ward, M., \& McGee, H. (2012). Latent structure of the Hospital Anxiety And Depression Scale: A 10-year systematic review. Journal of Psychosomatic Research, 72(3), 180-184. http://dx.doi.org/10.1016/ j.jpsychores.2011.06.008

Dancey, C. P. \& Reidy, J. (2006). Estatística sem matemática para psicologia usando SPSS para Windows. Porto Alegre, RS: Artmed.

Del Porto, J. A. (1999). Conceito e diagnóstico. Revista Brasileira de Psiquiatria, 21(1), 6-11. http://dx.doi.org/ 10.1590/S1516-44461999000500003

Elliott, R., Fox, C. M., Beltyukova, S. A., Stone, G. E., Gunderson, J., \& Zhang, X. (2006). Deconstructing therapy outcome measurement with Rasch analysis: The SCL-90-R. Psychological Assessment, 18, 359-372. http://dx.doi.org/ 10.1037/1040-3590.18.4.359

Ferster, C. B., Culbertson, S., \& Boren, M. C. (1977). Depressão clínica. Em C. B. Ferster, S. Culbertson, \& M. C. Boren, Princípios do Comportamento (pp. 699-725). São Paulo: Hucitec.
Gabriela da Silva Cremasco e Makilim Nunes Baptista

George, D. \& Mallery, P. (2003). SPSS for Windows step by step: A simple guide and reference. 11.0 update (4th ed.). Boston: Allyn \& Bacon.

Hung, M., Bounsanga, J., Tang, P., Chen, W., \& Cheng, C. (2015). The Factor Structure of the Hospital Anxiety and Depression Scale in Orthopedic Trauma Patients. Journal of Clinical Medicine Research, 7(6), 453-459. https:// doi.org/10.14740/jocmr2140w

Katon, W. J. (2003). Clinical and Health Services Relationships Between Major Depression, Depressive Symptoms, and General Medical Illness. Biological Psychiatry. 54(3), 216-226. https://doi.org/10.1016/ S0006-3223(03)00273-7

Linacre, J. M. (2010). A user's guide to Winsteps, Program Manual 3.70.0. Chicago: Winsteps.com

Linacre, J. M., \& Wright, B. D. (1991). WINSTEPS - RaschModel computer programs. Chicago: MESA Press.

Lindwall, M., Barkoukis, V., Grano, C., Lucidi, F., Raudsepp, L., Liukkonen, J., \& Thøgersen-Ntoumani, C. (2012). Method effects: The problem with negatively versus positively keyed items. Journal of Personality Assessment, 94(2), 196-204. $10.1080 / 00223891.2011 .645936$ http://dx.doi.org/

Lorenzo-Seva, U., \& Ferrando, P. J. (2006). FACTOR: A computer program to fit the exploratory factor analysis model. Behavioral Research Methods, 38(1), 88-91. http:// dx.doi.org/10.3758/BF03192753

Lorenzo-Seva, U., Timmerman, M. E., \& Kiers, H. A. L. (2011). The Hull method for selecting the number of common factors. Multivariate Behavioral Research, 46(2), 340-364. http://dx.doi.org/10.1080/00273171.2011.56452

Marques, M. A. B. (2016). Sintomas da depressão após lesão encefálica: Evidências de validade para EBADEP-HOSP$A M B$. (Tese de Doutorado, Universidade São Francisco, Itatiba, S. P.). Recuperado de https://www.usf.edu.br/

Messias, C. R. (2014). Sintomatologia Depressiva e Associações com Dor Crônica, Saúde Geral e Suporte Social em Pacientes Fibromiálgicos e Oncológicos (Dissertação de mestrado, Universidade São Francisco, Itatiba, S.P.) Recuperado de http://www.usf.edu.br

Organização Mundial da Saúde (OMS). (1993). Classificação dos transtornos mentais e do comportamento - CID-10: descrições e diretrizes diagnósticas. (10a ed.). Porto Alegre: Artes Médicas.

Pasquali, L. (2007). Validade dos testes psicológicos: Será possível reencontrar o caminho? Psicologia: Teoria e Pesquisa, 23(n.esp), 99-107. http://dx.doi.org/10.1590/ S0102-37722007000500019

Razavi, D., Delvaux, N., Farvacques, C., \& Robaye, E. (1990). Screening for adjustment disorders and major depressive disorder in cancer patients: a clinical model. British Journal of Psychiatry, 156(1), 79-83. http://dx.doi.org/ 10.1192/bjp.156.1.79 


\section{-4: INTERACÃO EM IT PSICOLOGIA}

Richards, D. (2011). Prevalence and clinical course of depression: A review. Clinical Psychology Review 31(7) 1117-1125. http://dx.doi.org/10.1016/j.cpr.2011.07.004.

Rodgers, J., Martin, C. R., Morse, R. C., Kendell, K., \& Verril, M. (2005). An investigation into the psychometric properties of the Hospital Anxiety and Depression Scale in patients with breast cancer. Health and Quality of Life Outcomes, 3, 41-53. http://dx.doi.org/10.1186/1477-7525-3-41.

Santor, D. A., Gregus, M., \& Welch, A. (2006). Eight Decades of Measurement in Depression. Measurement, 4(3), 135-155. Recuperado de http://www.scalesandmeasures.net/files/ files/Santor\%20et\%20al_\%20(2006)\%20Eight\%20Decades $\% 20(1)$.pdf

Serafini, A. J., Budzyn, C. S., \& Fonseca, T. L. R. (2017). Tipos de testes: Características e aplicabilidade. Em M. R. C. Lins, \& J. C. B. Borsa. Avaliação Psicológica: Aspectos teóricos e práticos (pp. 56-75). Rio de Janeiro: Editora Vozes.

Sette, C. P., Capitão, C. G., \& Carvalho, L. F. (2016). Depressive symptoms in patients with cancer. Open Journal of Medical Psychology, 5(1), 7-16. http://dx.doi.org/ojmp. 2016.51002.

Thompson, C. Affective disorders. (1989). Em C. Thompson. The Instruments of Psychiatric Research (pp. 87-126). London: John Wiley \& Sons Ltd.
Gabriela da Silva Cremasco e Makilim Nunes Baptista

Timmerman, M. E., \& Lorenzo-Seva, U. (2011). Dimensionality Assessment of Ordered Polytomous Items with Parallel Analysis. Psychological Methods, 16(2), 209-220. http:// dx.doi.org/10.1037/a0023353

Urbina, S. (2007). Fundamentos da Testagem Psicológica. Porto Alegre: Artes Médicas.

Velicer, W. F. (1976). Determining the number of components from the matrix of partial correlations. Psychometrika, 41(3), 321-327. http://dx.doi.org/10.1007/BF02293557

World Health Organization (WHO) (2017). Depression and other common mental disorders: Global health estimates. Recuperado de http://www.who.int/mental_health/ management/depression/ prevalence_global_health_estimates/en/

Data de submissão: 26/09/2017 Primeira decisão editorial: 24/11/2017 Aceite em 10/01/2018 